\title{
The Spark of Fourier Matrices: Connections to Vanishing Sums and Coprimeness और
}

\author{
Hema K. Achanta ${ }^{\mathrm{e}}$, Sampurna Biswas ${ }^{\mathrm{g}}$, Bhanumati N. Dasgupta ${ }^{\mathrm{f}}$, Soura \\ Dasgupta $^{\mathrm{h}, *}$, Mathews Jacob ${ }^{\mathrm{g}}$, Raghuraman Mudumbai ${ }^{\mathrm{g}}$ \\ ${ }^{e}$ Department of Systems Engineering, The University of Texas at Dallas, 800 W. Campbell Road, \\ Richardson, Texas 75080-3021. hema999achanta@ gma i l . com \\ ${ }^{f}$ Department of Mathematics, University of Iowa, Iowa City, IA 52242. \\ bhanumati@msn. com \\ ${ }^{g}$ Department of Electrical and Computer Engineering, University of Iowa, Iowa City, IA 52242. \\ sampurnakgp@gmail.com, mathews-jacob@uiowa.edu, \\ rmudumbai @ui owa. edu. \\ ${ }^{h}$ Department of Electrical and Computer Engineering, University of Iowa, Iowa City, IA 52242, \\ and Shandong Computer Science Center (National Supercomputer Center in Jinan), Shandong \\ Provincial Key Laboratory of Computer Networks. \\ dasgupta@engineering.uiowa.edu
}

\begin{abstract}
We consider conditions under which $L$ rows of an $N$ point DFT matrix form a matrix with spark $L+1$, i.e. a matrix with full spark. A matrix has spark $N+1$ if all $L$ columns are linearly independent. This has application in compressed sensing for MRI and synthetic aperture radar, where measurements are under sampled Fourier measurements, and the observation matrix comprises certain rows of the DFT matrix. It is known that contiguous rows of the DFT matrix render full spark and that from such a base set one can build a suite of other sets of rows that maintain full spark. We consider an alternative base set of the form $\{0,1, \cdots, K\} \backslash\{n\}$, and derive conditions on $K, n$ and the prime factors of $N$, under which full spark is retained. We show that such a matrix has full spark iff there are no $K$ distinct $N$-th roots of unity whose $n$-products form a vanishing sum, and leverage recent characterizations of vanishing sums of $N$-th roots of unity to establish the stated conditions.
\end{abstract}

Keywords:

Coprime sensing, full spark, compressed sensing, vanishing sums.

*Corresponding Author

Preprint submitted to Digital Signal Processing

April 24, 2016

(C) 2016. This manuscript version is made available under the Elsevier user license 


\section{Introduction}

The pioneering work of Pal and Vaidyanathan, [1], has introduced the notion of coprime sampling and revealed its importance to array processing in general and compressed sensing (CS) in particular. Broadly viewed [1] and the subsequent literature exploits certain fundamental properties of coprime integers. Inspired by these results, we consider yet another consequence of coprime conditions: their implication to the spark or Kruskal rank of a submatrix of the Discrete Fourier Transfrom (DFT) matrix.

The practical applications motivating our work include MRI [2, 3, 4] and synthetic aperture radar $[5,6,7]$, where under-sampled Fourier measurements are used to reconstruct images. In MRI in particular such observations are expensive and need to be kept to a minimum. It is of fundamental importance in such a case to know which Fourier sampling patterns are effective in image recovery and which are not, and how to keep these samples as small as possible, without impairing the reliability of recovery. Accordingly, this paper is dedicated to provide certain sampling patterns that permit such recovery and providing others that do not.

To be specific the vectorized images in such applications are often sparse and are observed through observation matrices that comprise submatrices of Discrete Fourier Transform (DFT) matrices obtained by removing several rows. The rows that are retained define the sampling patterns we seek. The spark or the Kruskal rank of a matrix is the smallest number of linearly dependent columns in the matrix. It plays a fundamental role in providing the answers we seek.

Thus consider the recovery of a $k$-sparse vector $x \in \mathbb{C}^{N}$ from its measurements

$$
y=A x,
$$

where a $k$-sparse vector is one which has at most $k$ nonzero entries. In this case it is possible to recover $x$ from $y$ even if the observation matrix $A \in \mathbb{C}^{L \times N}$, $L<N$ is fat. Should $\operatorname{spark}(A)>2 k[8,9]$ then in principle $x$ can be uniquely recovered from $y$, though the recovery may require a combinatorically involved $\ell_{0}$, constrained optimization. Under the so called Restricted Isometry Property (RIP), [10],[11] recovery of $x$ is possible using a convex, constrained $\ell_{1}$ optimization. A necessary and sufficient condition for such $\ell_{1}$ recovery is that the matrix $A$ satisfy the so called Null Space Condition (NSP), [12]. Though $\operatorname{spark}(A)>2 k$ by it self may not satisfy RIP or NSP there are loose relationships between spark and RIP in NSP, [12] that permit one to determine a higher spark level for which $\ell_{1}$ recovery is possible. 
Whatever level of spark is desirable it is important that it be achieved with the smallest number of measurements,i.e. with an observation matrix with the fewest number of rows. This is so as each extra measurement comes with a penalty (e.g. acquisition time in MRI). Thus it is our goal to provide conditions under which a matrix $A$ obtained by removing rows of a DFT matrix, has full spark: A matrix with $L$ rows has full spark if $\operatorname{spark}(A)=L+1$.

Since the computation of the spark of a large matrix is intractable, most designs of high spark matrices use the Welch bound $\operatorname{spark}(A)>1 / M$, where $M$ is the maximum off-diagonal entry of $A^{H} A$, a number known as the mutual coherence of $A$ [8]. Equiangular tight frame matrices achieve the Welch bound [13, 14].

As can be imagined, the Welch bound is highly conservative for DFT matrices. Many results on spark involve observation matrices whose elements are drawn from Gaussian distributions. Use of such observation matrices in applications like MRI and SAR is simply impractical. While the common practice in CS-MRI is to randomly sample the Fourier transform using a variable density sampling pattern, there are no theoretical guarantees on the recovery using these matrices. Recently, some researchers have shown that the partial Fourier matrices, obtained by picking the rows of the Discrete Fourier Transfrom (DFT) matrix, according to a particular difference set attains the Welch bound $[15,16]$.

In this paper we ask the following question: Which $L$ rows of an $N \times N$ DFT matrix yield a submatrix that is full spark? It is well known that $L$-consecutive rows of the DFT matrix have this property. Further [17] shows that should $L$ rows indexed by the set $\mathcal{M}$ have spark $L+1$, then so do those indexed by (a) a translation, (b) or the complement of $\mathcal{M}$, or (c) rows indexed by $M \mathcal{M}$ if $M$ is coprime with $N$.

Thus, starting from a base set comprising consecutive rows, one can generate a whole suite of row indices that yield full spark matrices. The question we address here is what happens if the base set is changed to a set of consecutive rows with one row missing? We argue that this augmented by (a-c) above yields a further formidable suite of full spark matrices.

A counter-example in [17] shows that such a base set need not yield a full spark matrix. We first provide a necessary and sufficient condition when the base set in focus here provides full spark matrices. With the spark depending on $n$, the location of the missing row that prevents contiguity, and $K$ is the total number of rows that are retained, this condition involves whether or not there are $K$ distinct $N$-th roots of unity, whose $n$-products sum to zero, i.e. constitute a vanishing sum. As the example of [17] involves $N=10$, a product of two prime numbers, 2 and 5 , we also separately consider the case where $N$ is the product of two primes and 
leverage recent results by [18] and [19], to give conditions on these prime factors, $K$ and $n$ that guarantee full spark, and as importantly those that do not. These conditions in turn depend on whether certain integers can be expressed as a linear combination of the prime factors of $N$. Details are provided in the bullets below together with contrasts with a preliminary version of this paper [22], that appeared in a conference proceedings and has terse proofs, fewer results and two important mistakes that we correct here.

(A) For a general $N$, we show that full spark obtains whenever $\left(\begin{array}{l}K \\ n\end{array}\right)$ is not a nonnegative integer combination of the prime factors of $N$, i.e. should these prime factors be $p_{i}$, then for all nonnegative integers $\alpha_{i}$

$$
\left(\begin{array}{l}
K \\
n
\end{array}\right) \neq \sum_{i} \alpha_{i} p_{i} .
$$

(B) Observe (A) provides a condition that is sufficient but not necessary. We show that should the missing index $n$ be either 1 or $K-1$, then in fact a necessary and sufficient condition can be found for full spark: that at least one among $K$ or $N-K$ not be a nonnegative integer combination of the prime factors of $N$. This corrects and strengthens the erroneous assertion in [22] that in this case a necessary and sufficient condition for full spark is that $K$, not be a nonnegative integer combination of the prime factors of $N$.

(C) The rest of the paper refines these when $N=p q$ is the product of two distinct primes $p$ and $q$. The conference paper incorrectly asserts that in such a case a necessary and sufficient condition for full spark is that $K$ not be a nonnegative integer combination of the prime factors $p$ and $q$, irrespective of the missing index $n$. In fact, at this point for $n \notin\{1, K-1\}$ a necessary and sufficient condition is not known. In a new set of results we explore the tightness of the sufficient condition in (1.1) and provide a much stronger condition even when $n \in\{1, K-1\}$.

(i) We show that in this case when $n \in\{1, K-1\}$, (B) can be vastly strengthened. That a necessary and sufficient condition for full spark is that $K$ be neither a multiple of $p$ nor of $q$. Observe this is much easier to check than checking whether $K$ and $N-K$ are nonnegative integer combinations of $p$ and $q$.

(ii) We next show that (1.1) is not in fact necessary. 
(iii) Just as providing conditions under which full spark is important, so is it important to provide conditions under which full spark cannot hold. Such conditions indicate which sampling patterns should be avoided. In this spirit we show that full spark does not hold if any among the integers $\{K, K-1, \cdots, K-n+1\}$ is a multiple of either $p$ or $q$.

The rest of the paper is structured as follows. Section 2 recounts relevant results, refines a result of [17], and sets the context of the remaining paper. Section 3 establishes the connection between spark and vanishing sums. Section 4 recounts recent characterizations of vanishing sums of roots of unity. Section 5 applies these to the spark problem. Section 6 provides conditions for which the full spark condition fails to hold. We observe that only one theorem presented in Section 5 and Section 6 is in [22]. Section 7 provides simulations. Section 8 is the conclusion.

\section{Context and Problem Statement}

Our observation matrices are submatrices of the $N \times N$ DFT matrix $W_{N}$. Specifically, with row and column indices taking values from the set

$$
\mathbb{Z}_{N}=\{0,1, \cdots, N-1\},
$$

the $i l$-th element of $W_{N}$ is $e^{\frac{j 2 \pi i l}{N}}$. These are of course $N$-th roots of unity. We will assume that the observation matrix $A(\mathcal{M}, N)$ only retains the rows of $W_{N}$ that are indexed by $\mathcal{M} \subset \mathbb{Z}_{N}$. The central question of this paper is: What conditions on $\mathcal{M}$ and $N$ ensure that $A(\mathcal{M}, N)$ has full spark? We now recount some important results addressing this question.

Suppose for some $i$ and $\mu, \mathcal{M}=\{i, i+\mu, \cdots, i+\mu-1\}$ i.e. contains $\mu$ consecutive elements of $\mathbb{Z}_{N}$. Define

$$
z_{n}=e^{j \frac{2 \pi n}{N}}
$$

Then the matrix comprising any $l$ columns of $A(\mathcal{M}, N)$, indexed by the integers $i_{1}, \cdots, i_{\mu}$ comprises contiguous rows of $W_{N}$, and can be expressed as

$$
\left[\begin{array}{cccc}
1 & 1 & \cdots & 1 \\
z_{i_{1}} & z_{i_{2}} & \cdots & z_{i_{\mu}} \\
\vdots & \vdots & \vdots & \vdots \\
z_{i_{\mu}}^{\mu-1} & z_{i_{2}}^{\mu-1} & \cdots & z_{i_{\mu}}^{\mu-1}
\end{array}\right] \operatorname{diag}\left\{z_{i_{1}}^{i}, z_{i_{2}}^{i}, \cdots, z_{i_{\mu}}^{i}\right\}
$$


and being a product of a Vandermonde and a nonsingular diagonal matrix, is thus nosingular for distinct $z_{i_{n}}$. Thus, this $A(\mathcal{M}, N)$ has full spark. A less obvious result can be traced back to Chebotarëv in the early 20th century, (see [20]).

Theorem 2.1. Suppose $N$ is prime. Then for all $\mathcal{M} \subset \mathbb{Z}_{N}, A(\mathcal{M}, N)$ has full spark.

The most sophisticated results are in [17] and provide a springboard for the results of this paper. The one motivating us is a set of sufficient conditions.

Theorem 2.2. Suppose for some $\mathcal{M} \subset \mathbb{Z}_{N}, A(\mathcal{M}, N)$ has full spark. Then so does:

(i) $A((\mathcal{M}+i) \bmod N, N)$ for all $i \in \mathbb{Z}_{N}$, i.e. the full spark condition is preserved under all translations.

(ii) $A(M \mathcal{M}, N)$ for all $M$ that is coprime with $N$.

(iii) $A\left(\mathbb{Z}_{N} \backslash \mathcal{M}, N\right)$.

This theorem permits one to build entire suites of $\mathcal{M}_{i}$ for which $A\left(\mathcal{M}_{i}, N\right)$ is full spark, from any $\mathcal{M}$ for which $A(\mathcal{M}, N)$ is full spark. Consider the following example.

Example 2.1. Consider $N=10$, and $\mathcal{M}=\{0,1,2\}$. Then using Theorem 2.2, matrices obtained by retaining just the following sets of rows of the DFT matrix have full spark: $\{0,3,6\},\{2,5,8\}$ and $\{0,1,3,4,6,7,9\}$.

In fact this result has been strengthened in [23].

Theorem 2.3. [23] Suppose for positive integers $M, K, \mathcal{M}=\{i, i+M, i+2 M$, $\cdots, i+(K-1) M\}$, with $i+(K-1) M<N$. Then $A(\mathcal{M}, N)$ has full spark iff $M$ and $N$ are coprime. Further if $A(\mathcal{M}, N)$ does not have full spark, then $\operatorname{spark}(A)=2$.

As illustrated by Example 2.1 starting from the base set $\mathcal{M}=\mathbb{Z}_{K+1}$, the full spark nature of $A(\mathcal{M}, N)$ permits the construction of a plethora of subsets of $\mathbb{Z}_{N}$ that yield the full spark property. The hallmark of $\mathcal{M}=\mathbb{Z}_{K+1}$ is that consecutive rows of $W_{N}$ comprise the observation matrix. The rest of the paper asks: What if a single frame from $\{0,1, \cdots, K\}$ is missing? What conditions guarantee full spark observation matrices? These sufficient conditions, together with Theorem 2.2 then generate a rich class of further row indices that guarantee full spark observations. 
To fix notations, the base sets we will consider will be for some $K \in \mathbb{Z}_{N}$ $\{N-1\}$ and $m \in \mathbb{Z}_{K} \backslash\{0\}$,

$$
\mathcal{S}(K, m)=\mathbb{Z}_{K+1} \backslash\{m\}
$$

i.e. all integers from 0 to $K$ excepting $m$. Theorem 2.2 then permits even the base set $\mathcal{S}(K, 1)$ to be converted into sets that may have more than just one element missing. For example, consider $N=8, K=4$, and the base set $\{0,2,3,4\}$. Using (i), (iii) of Theorem 2.2, one can deduce whether the submatrices of $W_{10}$, comprising rows indexed by the sets $\{1,5,6,7\}$ and $\{0,4,5,6\}$ have full spark. Effectively, the second of these is a base set missing three instead of a solitary element.

That this problem is nontrivial is gauged by the following example from [17]. Our results directly explain this example.

Example 2.2. Consider $N=10, \mathcal{M}=\{0,1,3,4\}$. Then $A(\mathcal{M}, 10)$ does not have full spark as its columns indexed by the set $\{0,1,2,6\}$ are linearly dependent.

\section{A Necessary and Sufficient Condition: Role of Vanishing Sums}

This section establishes an equivalence between the full spark nature of $A(\mathcal{S}(K, n), N)$ and the existence of certain vanishing sums of $N$-th roots of unity. Consider a set of $N$-th roots of unity

$$
\mathcal{W}=\left\{w_{1}, w_{2}, \cdots, w_{K}\right\}
$$

We say these form a vanishing sum if

$$
\sum_{i=1}^{K} w_{i}=0
$$

In the sequel we will refer to the sum of $m$-products of a set of complex numbers. This is the sum of the products of the elements belonging to all subsets of the set with cardinality $m$. For example, the sum of 2-products of $\left\{a_{1}, a_{2}, a_{3}\right\}$ is $a_{1} a_{2}+a_{1} a_{3}+a_{2} a_{3}$. We will denote $\Sigma(\mathcal{W}, m)$ to be the sum of the $m$-products of the elements of $\mathcal{W}$, with

$$
\Sigma(\mathcal{W}, 0)=1
$$


Of course the sum of 1-products of the elements of $\left\{a_{1}, a_{2}, a_{3}\right\}$ is $a_{1}+a_{2}+a_{3}$. Thus

$$
a_{1} a_{2}+a_{1} a_{3}+a_{2} a_{3}=\Sigma\left(\left\{a_{1}, a_{2}, a_{3}\right\}, 2\right) .
$$

Before presenting and proving the main theorem we illustrate the basic idea with Example 2.2. Consider four columns of $A(\mathcal{M}, 10)$, where as in Example 2.2, $\mathcal{M}=\mathcal{S}(4,2)$. The matrix does not have full spark if and only if it has four linearly dependent columns. Consider the matrix formed by such a set of four columns, i.e. for suitable distinct $z_{i}, z_{k}, z_{l}, z_{n}$ as in (2.2)

$$
\left[\begin{array}{cccc}
1 & 1 & 1 & 1 \\
z_{i} & z_{k} & z_{l} & z_{n} \\
z_{i}^{3} & z_{k}^{3} & z_{l}^{3} & z_{n}^{3} \\
z_{i}^{4} & z_{k}^{4} & z_{l}^{4} & z_{n}^{4}
\end{array}\right]
$$

As this matrix is singular there exists a nonzero $\theta=\left[\theta_{0}, \theta_{1}, \theta_{3}, \theta_{4}\right]^{T}$ such that

$$
\theta^{T}\left[\begin{array}{cccc}
1 & 1 & 1 & 1 \\
z_{i} & z_{k} & z_{l} & z_{n} \\
z_{i}^{3} & z_{k}^{3} & z_{l}^{3} & z_{n}^{3} \\
z_{i}^{4} & z_{k}^{4} & z_{l}^{4} & z_{n}^{4}
\end{array}\right]=\left[\begin{array}{llll}
\theta\left(z_{i}\right) & \theta\left(z_{k}\right) & \theta\left(z_{l}\right) & \theta\left(z_{n}\right)
\end{array}\right]=0
$$

where

$$
\theta(z)=\theta_{0}+\theta_{1} z+\theta_{3} z^{3}+\theta_{4} z^{4} .
$$

Thus lack of full spark is equivalent to the existence of a nonzero polynomial as in (3.4) that has the four $N$-th roots of unity $z_{i}, z_{k}, z_{l}, z_{n}$. Observe, $\theta(z)$ in (3.4) has the degree two coefficient zero. This coefficient is the sum of the 2-products of $\left\{z_{i}, z_{k}, z_{l}, z_{n}\right\}$. As the polynomial has degree four these are the only roots of this polynomial, there thus holds:

$$
z_{i} z_{k}+z_{i} z_{l}+z_{i} z_{n}+z_{k} z_{l}+z_{k} z_{n}+z_{l} z_{n}=0 .
$$

As each column corresponds to a distinct $N$-th root of unity, and all columns are present and between then account for all $N$-th roots of unity, we have effectively shown for this example that lack of full spark is equivalent to the existence of four $N$-th roots of unity whose 2-products form a vanishing sum. Observe that this argument critically relies on the fact that only one row of the first five are missing. Also observe that missing coefficient in $\theta(z)$ corresponds to the index of the missing row among the first five. Further each summand in (3.5) is itself an 
$N$-th root of unity. Since four distinct columns of the DFT matrix are involved $z_{i}$, $z_{k}, z_{l}, z_{n}$ are distinct. Their 2-products though need not be.

Generalizing this result leads to the following promised equivalence.

Theorem 3.1. For integers $1 \leq n<K<N, A(\mathcal{S}(K, n), N)$, see (2.3), does not have full spark iff there exist $K$ distinct $N$-th roots of unity whose $n$-products form a vanishing sum.

Proof. Proof of Sufficiency: Suppose there are $K$ distinct $N$-th roots of unity, $z_{n_{1}}, \cdots, z_{n_{K}}$ whose $n$-products form a vanishing sum. Consider the polynomial

$$
\prod_{i=1}^{K}\left(z-z_{n_{i}}\right)=\sum_{l=0}^{K} g_{i} z^{i}=g(z)
$$

Observe $g_{n}$ is the sum of $n$-products of the roots of $g(z)$ and hence by definition of vanishing sums, is zero. Define the vector

$$
G=\left[\begin{array}{llllll}
g_{0} & \cdots & g_{n-1} & g_{n+1} & \cdots & g_{K}
\end{array}\right]^{T} \in \mathbb{C}^{K} .
$$

With $z_{n}$ defined in (2.2) define

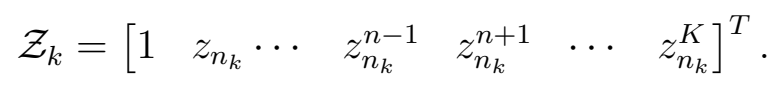

As the row indexed by $n$ is missing from the other contiguous rows that are retained in $A(\mathcal{S}(K, n), N), \mathcal{Z}_{1}, \cdots, \mathcal{Z}_{K}$ comprise $K$-columns of $A(\mathcal{S}(K, n), N)$. From (3.6)

$$
G^{T}\left[\mathcal{Z}_{1}, \cdots, \mathcal{Z}_{K}\right]=0
$$

i.e. this submatrix of $A(\mathcal{S}(K, n), N)$ has rank less than $K$, proving that $A(\mathcal{S}(K, n), N)$ has spark less than $K+1$.

Proof of Necessity: Since it has $K$ rows, $A(\mathcal{S}(K, n), N)$ does not have full spark only if it has $K$ distinct columns that are linearly dependent. Suppose these columns are indexed from the set $\left\{n_{1}, \cdots, n_{K}\right\} \subset \mathbb{Z}_{N}$. Then there is a nonzero $G \in \mathbb{C}^{K}$ such that under (3.7)

$$
G^{T} \mathcal{Z}_{k}=g\left(z_{n_{k}}\right)=0
$$

As $g(z)$ has degree $K, z_{n_{1}}, \cdots, z_{n_{K}}$ are the only roots of this polynomial. Further by definition of $A(\mathcal{S}(K, n), N)$ the coefficient of $z^{n}$ in $g(z)$ is zero. As this coeffi- 
cient is the sum of the $n$ products of the distinct roots $z_{n_{1}}, \cdots, z_{n_{K}}$, the $n$-products of these roots form a vanishing sum.

In subsequent sections we refine this result to pinpoint conditions on just $K, N$ and $n$ under which the stated vanishing sums exist. But first we have an immediate consequence of Theorem 3.1.

Theorem 3.2. For integers $1 \leq n<K<N$ and $\mathcal{S}(K, n)$ defined in (2.3), $A(\mathcal{S}(K, n), N)$ has full spark iff $A(\mathcal{S}(K, K-n), N)$ has full spark.

Proof. From Theorem 3.1, $A(\mathcal{S}(K, n), N)$ does not have full spark iff there are $K$ distinct $N$-th roots of unity whose $n$ products form a vanishing sum. Suppose these are $w_{1}, \cdots, w_{K}$. In other words $\Sigma\left(\left\{w_{1}, \cdots, w_{K}\right\}, n\right)=0$. Consequently as the $w_{i}$ are all nonzero

$$
\frac{\Sigma\left(\left\{w_{1}, \cdots, w_{K}\right\}, n\right)}{\prod_{i=1}^{K} w_{i}}=0 .
$$

Consider the product of any $n$ of these roots, without loss of generality

$$
\prod_{i=1}^{n} w_{i}
$$

As $w_{i}$ are on the unit circle $1 / w_{i}=w_{i}^{*}$. Thus

$$
\begin{aligned}
\frac{\prod_{i=1}^{n} w_{i}}{\prod_{i=1}^{K} w_{i}} & =\frac{1}{\prod_{i=n+1}^{K} w_{i}} \\
& =\left(\prod_{i=n+1}^{K} w_{i}\right)^{*}
\end{aligned}
$$

which is the conjugate of the product of the $K-n$ roots that are not in the set $\left\{w_{1}, \cdots, w_{n}\right\}$. Thus the sum of $n$-products of the roots in $w_{1}, \cdots, w_{K}$ is zero iff the conjugate of the sum of $(K-n)$-products of these very roots is zero. Thus the $n$-products form a vanishing sum iff the $(K-n)$-products do. The result follows.

Thus ascertaining whether $A(\mathcal{S}(K, 1), N)$ has full spark, also ascertains whether $A(\mathcal{S}(K, K-1), N)$ has full spark, amplifying the virtues of just considering the base set $\mathcal{S}(K, 1)$. 


\section{Characterization of Vanishing Sums}

The previous section squarely tied the full spark property of the base set $\mathcal{S}(K, n)$ to whether there are $K$ distinct $N$-th roots of unity whose $n$-products form a vanishing sum. We now recount the seminal result of Lam and Leung, [18] and its subsequent refinement, [19]. Lam and Leung ask and answer the question: Given a number $r$, when are there $r, N$-th roots of unity, not necessarily distinct, that form a vanishing sum? In a subsequent paper, [19] answers the questions when these root are mandated to be distinct. Both results tie the answer to the prime factors of $N$. In the sequel we define $\mathbb{N}$ to be the set of all nonnegative integers.

Before providing these characterizations let us examine an example. Suppose $N=6$. Its prime factors are 2 and 3 . Consider the set

$$
S_{2}(x)=x+2 \mathbb{Z}_{3}=\{x, x+2, x+4\} .
$$

Clearly for any

$$
\begin{aligned}
\sum_{s_{i} \in S_{2}(x)} e^{j 2 \pi \frac{s_{i}}{6}} & =e^{j 2 \pi \frac{x}{6}}\left(1+e^{j 2 \pi \frac{2}{6}}+e^{j 2 \pi \frac{4}{6}}\right) \\
& =e^{j 2 \pi \frac{x}{6}}\left(1+e^{j \frac{2 \pi}{3}}+e^{j \frac{4 \pi}{3}}\right) \\
& =0 .
\end{aligned}
$$

Similarly, with

$$
\begin{gathered}
S_{3}(y)=y+3 \mathbb{Z}_{2}=\{y, y+3\}, \\
\sum_{s_{i} \in S_{3}(y)} e^{j 2 \pi \frac{s_{i}}{6}}=0 .
\end{gathered}
$$

Equally

$$
\sum_{s_{i} \in S_{3}(y) \bigcup S_{2}(x)} e^{j 2 \pi \frac{s_{i}}{6}}=0 .
$$

In fact Lam and Leung show that a set of 6-th roots of unity $\left\{e^{j 2 \pi \frac{s_{i}}{6}}\right\}_{i=1}^{r}$ sum to zero iff they can be separated into cosets such as $(4.8,4.9)$. This is equivalent to saying that $r$ their number is for nonnegative integers $\alpha$ and $\beta, 2 \alpha+3 \beta$. In other words $r \in 2 \mathbb{N}+3 \mathbb{N} ; 2$ and 3 are of course the prime factors of 6 .

We now formally state the Lam and Leung theorem. 
Theorem 4.1. [18] Suppose $N>1$ is a positive integer, with prime factors $p_{1}, \cdots p_{l}$. Then there exist $r$ possibly nondistinct $N$-th roots of unity $\left\{w_{i}\right\}_{i=1}^{r}$ that form a vanishing sum iff

$$
r \in \sum_{i=1}^{l} p_{i} \mathbb{N} .
$$

In other words $r$ is a nonnegative integer combination of the prime factors of $N$. Sivek, [19], extends Theorem 4.1 to distinct $N$-th roots of unity. The intuition behind this result is as follows. As there are precisely $N$ distinct $N$-th roots of unity, and these themselves add to zero, $r$ distinct $N$-th roots of unity form a vanishing sum iff the remaining $N-r$ do so as well.

Theorem 4.2. [19] Suppose $N>1$ is a positive integer, with prime factors $p_{1}, \cdots p_{l}$. Then there exist $r$ distinct $N$-th roots of unity $\left\{w_{i}\right\}_{i=1}^{r}$ that form a vanishing sum iff

$$
r \in \sum_{i=1}^{l} p_{i} \mathbb{N} \text { and } N-r \in \sum_{i=1}^{l} p_{i} \mathbb{N}
$$

Though Lam and Leung provide a characterization of the $N$-th roots of unity that form a vanishing sum, this characterization is particularly clean when $N$ is a product of two primes $p$ and $q$, i.e. $N=p q$. To provide this characterization we introduce two types of cosets that generalize $(4.8,4.9)$. For some nonnegative integers $x$ and $y$

$$
S_{p}(x)=x+p \mathbb{Z}_{q}, x \in \mathbb{Z}_{q}
$$

and

$$
S_{q}(y)=y+q \mathbb{Z}_{p}, y \in \mathbb{Z}_{p} .
$$

In the sequel we will call (4.10) and (4.11) $p$ and $q$ cosets, respectively. The elements of $p$-cosets, for example have differences that are multiples of $p$. Then [18] shows that $r=\alpha p+\beta q, \alpha \in \mathbb{N}$ and $\beta \in \mathbb{N}, N$-th roots of unity

$$
\left\{e^{\frac{2 \pi j s_{i}}{N}}\right\}_{i=1}^{r}, s_{i} \in \mathbb{Z}_{N},
$$

form a vanishing sum iff the $s_{i}$ can be distributed across complete $\alpha$ complete $q$-cosets and $\beta$ complete $p$-cosets. Formally, we have the following theorem.

Theorem 4.3. [18] Consider the possibly, nondistinct $r, N$-th roots of unity in (4.12). Call the index set $\mathcal{I}=\{1, \cdots, r\}$. Then the roots in (4.12) form a vanishing sum iff $\mathcal{I}$ admits a partitioning $\mathcal{I}_{l}$ that has the following property. For a given 
l one of the following holds: either $\mathcal{I}_{l}$ has cardinality $p$ and for some nonnegative integer $x_{l}$,

$$
\left\{s_{i} \mid i \in \mathcal{I}_{l}\right\}=S_{p}\left(x_{l}\right)
$$

or $\mathcal{I}_{l}$ has cardinality q and for some nonnegative integer $y_{l}$,

$$
\left\{s_{i} \mid i \in \mathcal{I}_{l}\right\}=S_{q}\left(y_{l}\right) .
$$

For example, with $N=6$ and $r=5$, of the five indices, two of the $s_{i}$ 's must form a 3-coset and the remaining three must form a 2-coset. Further for every $x_{l}$ and $y_{l}$

$$
\sum_{s \in S_{p}\left(x_{l}\right)} e^{j \frac{2 \pi s}{N}}=\sum_{s \in S_{q}\left(y_{l}\right)} e^{j \frac{2 \pi s}{N}}=0 .
$$

\section{Translation to full spark}

We now apply the results on vanishing sum given in Section 4 to the full spark characterization of Section 3, beginning with two results for the base set is $\mathcal{S}(K, 1)$. The first is for general $N$. The second when $N$ is the product of only two distinct primes. We will explain later why we are treating the $\mathcal{S}(K, 1)$ case differently from $\mathcal{S}(K, n)$ for a general $n$.

Theorem 5.1. Suppose the prime factors of $N$ are $p_{1}, \cdots, p_{l}$. Consider for integer $1<K<N$, and $\mathcal{S}(K, 1)$ and $\mathcal{S}(K, K-1)$ defined in (2.3). Then $A(\mathcal{S}(K, 1), N)$ and $A(\mathcal{S}(K, K-1), N)$ do not have full spark iff

$$
K \in \sum_{i=1}^{l} p_{i} \mathbb{N} \text { and } N-K \in \sum_{i=1}^{l} p_{i} \mathbb{N} .
$$

Proof. From Theorem 3.1, $A(\mathcal{S}(K, 1), N)$ does not have full spark iff there are $K$ distinct $N$-th roots of unity that form a vanishing sum. Further from Theorem 3.2, $A(\mathcal{S}(K, 1), N)$ has full spark iff $A(\mathcal{S}(K, K-1), N)$ has full spark. Then the result follows from Theorem 4.2.

Thus if $N=5 \times 11 \times 13^{2}=9295, A(\mathcal{S}(8,1), 9295)$ and $A(\mathcal{S}(14,1), 9295)$ have full spark. Similarly for any $n>1 A\left(\mathcal{S}(K, 1), 2^{n}\right)$ has full spark iff $K$ is odd.

It turns out when $N$ is the product of just two prime numbers a much stronger result is possible. 
Theorem 5.2. Suppose $p$ and $q$ are distinct prime numbers $N=p q, 0<K<$ $N$ and $\mathcal{S}(K, 1)$ and $\mathcal{S}(K, K-1)$ defined in (2.3). Then $A(\mathcal{S}(K, 1), N)$ and $A(\mathcal{S}(K, K-1), N)$ do not have full spark iff either

$$
K \in p \mathbb{N} \text {. }
$$

or

$$
K \in q \mathbb{N} .
$$

Proof. Because of Theorem 3.2 it suffices to work with $A(\mathcal{S}(K, 1), N)$. In view of Theorem 5.1, there exist nonnegative integers $a, b, c, d$ such that

$$
K=a p+b q
$$

and

$$
N-K=c p+d q .
$$

As $K \in \mathbb{Z}_{N}, N-K \in \mathbb{Z}_{N}$, and $N=p q, a, c$ must be in $\mathbb{Z}_{p}$ and $b, d$ in $\mathbb{Z}_{q}$. To prove the "if part" suppose without loss of generality

$$
K=a p, a \in \mathbb{Z}_{q}
$$

Then

$$
\begin{aligned}
N-K & =p q-a p \\
& =(q-a) p
\end{aligned}
$$

As $q-a \in \mathbb{Z}_{q}, N-K \in p \mathbb{N}$ and the result holds.

To prove the "only if" part, observe that one obtains from (5.16) and (5.17) that

$$
p q=N=(a+c) p+(b+d) q .
$$

Further $a+c \in \mathbb{Z}_{q}$ and $b+d \in \mathbb{Z}_{p}$ as other wise the right hand side exceeds the left hand side. Further

$$
(q-(a+c)) p=(b+d) q
$$

As $p$ and $q$ are prime and $(a+c) \in \mathbb{Z}_{q}$ and $b+d \in \mathbb{Z}_{p}$, either $a+c=q$ and $b+d=0$ or $b+d=p$ and $a+c=0$. As $a, b, c, d$ are all nonnegative, either $b=d=0$ or $a=c=0$. 
Thus, for $N=77, A(\mathcal{S}(K, 1), N)$ and $A(\mathcal{S}(K, K-1), N)$ do not have full spark iff $K$ is either a multiple of 11 or 7 . However they have full spark for $K=11+7=18$ as it is not a multiple of either 7 or 11 .

We now turn to more general $n$.

Theorem 5.3. Suppose the prime factors of $N$ are $p_{1}, \cdots, p_{l}$. Consider for integer $1<n<K-1<N-1, \mathcal{S}(K, n)$ defined in (2.3). Then $A(\mathcal{S}(K, n), N)$ has full spark if

$$
\left(\begin{array}{l}
K \\
n
\end{array}\right) \notin \sum_{i=1}^{k} p_{i} \mathbb{N} .
$$

Proof. From Theorem 3.1, $A(\mathcal{S}(K, n), N)$ does not have full spark iff there are $K$ distinct $N$-th roots of unity whose $n$-products form a vanishing sum. Each $n$ product of $N$-th roots of unity is itself an $N$-th root of unity. Further there are $\left(\begin{array}{l}K \\ n\end{array}\right)$ such $n$-products. Then the result follows from Theorem 4.1.

Unlike Theorem 5.1, this theorem does not provide a necessary and sufficient condition. All that the violation of (5.18) guarantees is that there are $\left(\begin{array}{l}K \\ n\end{array}\right), N$-th roots of unity that form a vanishing sum. This does not necessarily imply that these can be expressed as the $n$-products of $K$ distinct $N$-th roots of unity. Indeed in Section 5.1, we show that $A(\mathcal{S}(5,2), 21)$ has full spark despite the fact that $\left(\begin{array}{l}5 \\ 2\end{array}\right)=10=7+3$ where 7 and 3 are prime factors of 21 .

It is also instructive that even though certain numbers may be distinct, their $n$-products need not be. Thus the additional condition imposed by Theorem 4.2 does not apply to $\left(\begin{array}{l}K \\ n\end{array}\right)$.

On the other hand, return to Example 2.2 in which $N=10, K=4$, and $n=2$. Clearly $\left(\begin{array}{l}4 \\ 2\end{array}\right)=6$ is even and hence a multiple of one of the prime factors of 10 . Thus this example violates the sufficient condition of Theorem 5.3. However, as Theorem 5.3 does not provide a sufficient condition it fails to provide a definitive explanation of the lack of full spark evinced by Example 2.2. That explanation is postponed to Section 6.

\subsection{The case of $\mathcal{S}(5,2)$ when $N=21$}

Consider the case flagged earlier where $N=p q, p=3, q=7$ and $K=5$. We will argue that no five distinct $N$-th roots of unity have 2-products that form a vanishing sum. Thus even though $\left(\begin{array}{c}K \\ 2\end{array}\right)=3+7, A(\mathcal{S}(5,2), 21)$ has full spark.

To establish a contradiction, suppose there are 5 distinct $s_{i}$ such that the 2products of

$$
e^{\frac{2 \pi j s_{i}}{21}}
$$


vanish. If these 2-products form a vanishing sum then so do the 2-products formed by

$$
e^{\frac{2 \pi j\left(s_{i}-t\right)}{21}}
$$

for any $t$. Thus without loss of generality assume one of the $s_{i}$ is zero and the set of $s_{i}$ can be chosen to be

$$
S=\left\{0, s_{1}, s_{2}, s_{3}, s_{4}\right\}
$$

As $\left(\begin{array}{l}5 \\ 2\end{array}\right)=10=p+q$, arguing as in the analysis of Example 2.2, from Theorem 4.3 , the 2 -sums of the $s_{i}$ must break into two cosets: For some $z_{i} \in \mathbb{Z}_{21}$

$$
S_{7}\left(z_{1}\right)=z_{1}+\{0,7,14\}
$$

and

$$
S_{3}\left(z_{2}\right)=z_{2}+\{0,3,6,9,12,15,18\} .
$$

In the sequel, we will drop the arguments $z_{i}$ and refer to these cosets as just $S_{3}$ and $S_{7}$. Now the 2-sums of the elements of $S$ include $T=\left\{s_{1}, s_{2}, s_{3}, s_{4}\right\}$. Consider the following cases.

Case I: All elements of $T$ are in the same coset: As $\left|S_{7}\right|=3, T \subset S_{3}$. Then, without loss of generality for distinct $\alpha_{i} \in \mathbb{Z}_{7}$

$$
s_{i}=s_{1}+3 \alpha_{i}, i \in\{2,3,4\} .
$$

Then every 2-sum takes the form for some nonnegative integers $\beta_{l}$

$$
t_{l}=2 s_{1}+3 \beta_{l}
$$

As three of these $t_{l}$ form $S_{7}$, without loss of generality $l \in\{1,2,3\}$, there holds:

$$
\begin{aligned}
7 & =t_{2}-t_{1} \\
& =3\left(\beta_{2}-\beta_{1}\right) .
\end{aligned}
$$

This cannot hold as 7 is a prime.

Case II: $\left\{s_{1}, s_{2}, s_{3}\right\} \subset S_{r}, r \in\{3,7\}$ but $s_{4} \notin S_{r}:$ We argue that $r=3$. To establish a contradiction suppose $\left\{s_{1}, s_{2}, s_{3}\right\} \subset S_{7}$. Arguing as above, without 
loss of generality for distinct $\alpha_{i} \in \mathbb{Z}_{3}$

$$
s_{i}=s_{1}+7 \alpha_{i}, i \in\{2,3\} \text {. }
$$

Further as $\left|S_{7}\right|=3, s_{4}+s_{l} \in S_{3}$ for all $l \in\{1,2,3\}$. Then $\left|s_{2}-s_{3}\right|$ is divisible by 3. From (5.24), $\left|s_{2}-s_{3}\right|$ is also divisible by 7 , i.e. is divisible by 21 . This cannot hold as $\left|s_{2}-s_{3}\right| \bmod 21 \neq 0$.

Thus $r=3$. Now $s_{4} \in S_{7}$. We assert first that for $\{i, k\} \subset\{1,2,3\}, S_{7} \neq$ $\left\{s_{4}, s_{4}+s_{i}, s_{4}+s_{k}\right\}$. This is so as in this case $\left|s_{i}-s_{k}\right|$ is divisible by 7 , while as $s_{i}$ and $s_{k}$ are both in $S_{3},\left|s_{i}-s_{k}\right|$ is also divisible by 3 , and $\left|s_{i}-s_{k}\right| \bmod 21=0$. Likewise, for $\{i, k, l\}=\{1,2,3\}, S_{7} \neq\left\{s_{4}, s_{k}+s_{i}, s_{l}+s_{k}\right\}$ as then $\left|s_{i}-s_{l}\right|$ $\bmod 21=0$. Thus, without loss of generality, there remain two possibilities. Either

$$
S_{7}=\left\{s_{4}, s_{4}+s_{1}, s_{1}+s_{2}\right\}
$$

or

$$
S_{7}=\left\{s_{4}, s_{4}+s_{1}, s_{3}+s_{2}\right\} .
$$

We will now eliminate both. By definition of $S_{7},(5.25)$ implies that $s_{1}$ is a nonzero multiple of 7. In particular,

$$
s_{1}=7 \alpha, \alpha \in\{1,2\} \text {. }
$$

Further, for some $z \in \mathbb{Z}_{7}$,

$$
\left\{s_{1}, s_{2}, s_{3}, s_{1}+s_{3}, s_{2}+s_{3}, s_{2}+s_{4}, s_{3}+s_{4}\right\}=z+3 \mathbb{Z}_{7} .
$$

Thus for distinct $\beta_{i} \in \mathbb{Z}_{7}$

$$
s_{2}=7 \alpha+3 \beta_{2} \text { and } s_{3}=7 \alpha+3 \beta_{3} \Rightarrow s_{2}+s_{3}=14 \alpha+3\left(\beta_{2}+\beta_{3}\right) .
$$

Thus $s_{2}+s_{3}-s_{1}=7 \alpha+3\left(\beta_{2}+\beta_{3}\right)$ is a multiple of 3 . This cannot happen unless $\alpha$ is a multiple of 3 , violating the fact that $\alpha \in\{1,2\}$.

Turn now to (5.26). Again (5.27) holds. In this case for some $z \in \mathbb{Z}_{7}$

$$
\left\{s_{1}, s_{2}, s_{3}, s_{1}+s_{3}, s_{1}+s_{2}, s_{2}+s_{4}, s_{3}+s_{4}\right\}=z+3 \mathbb{Z}_{7}
$$

and for some $\beta \in \mathbb{Z}_{7} \backslash\{0\}, s_{2}=3 \beta+7 \alpha$. Thus $s_{1}+s_{2}=14 \alpha+3 \beta$. As both 
$s_{1}+s_{2}$ and $s_{1}$ are in the set on the left hand side of (5.29)

$$
\begin{aligned}
s_{1}+s_{2}-s_{1} & =14 \alpha+3 \beta-7 \alpha \\
& =7 \alpha+3 \beta
\end{aligned}
$$

is a multiple of 3. Again this cannot happen as $\alpha \in\{1,2\}$.

Case III: $\left\{s_{1}, s_{2}\right\} \subset S_{7}$ and $\left\{s_{3}, s_{4}\right\} \subset S_{3}$ : Then for $\alpha \in\{1,2\}$,

$$
s_{2}=s_{1}+7 \alpha
$$

As $\left|S_{7}\right|=3$ at least three among $s_{1}+s_{3}, s_{1}+s_{4}, s_{2}+s_{3}$ and $s_{2}+s_{4}$ are in $S_{3}$. without loss of generality assume $s_{1}+s_{3}$ and $s_{2}+s_{3}$ are in $S_{3}$. Then $\left|s_{2}-s_{1}\right|$ is a multiple of 3. From (5.30) $\left|s_{2}-s_{1}\right|$ is also a multiple of 7. Again a contradiction obtains.

\section{Some conditions for lack of full spark}

Theorem 5.3 provides only a sufficient condition for $A(\mathcal{S}(K, n), N)$ to have full spark, when $1<n<K-1$. Section 5.1 proves by analyzing an example that this condition is not necessary. For the special case of $N=p q$, in this section we provide sufficient conditions under which $A(\mathcal{S}(K, n), N)$ does not have full spark. In view of Theorem 3.1 these sufficient conditions for lack of full spark will involve proving that $m$-products of certain distinct $N$-th roots of unity form vanishing sums.

Consider a set of numbers as in $(3.1), \Sigma(\mathcal{W}, m)$ the sum of its $m$-products. Observe that the following recursion obtains:

$$
m \Sigma(\mathcal{W}, m)=\sum_{i=1}^{K} w_{i} \Sigma\left(\mathcal{W} \backslash\left\{w_{i}\right\}, m-1\right)
$$

This is so as each $m$-product appears $m$ times in the right hand side of (6.1). Additionally, under (3.3), for any $n \in\{1, \cdots, K\}$

$$
\Sigma(\mathcal{W}, m)=w_{n} \Sigma\left(\mathcal{W} \backslash\left\{w_{n}\right\}, m-1\right)+\Sigma\left(\mathcal{W} \backslash\left\{w_{n}\right\}, m\right)
$$

Now suppose for some $l$

$$
\Sigma(\mathcal{W}, l)=0
$$


438

447

Then from (6.2), for any $n \in\{1, \cdots, K\}$, there holds

$$
\begin{aligned}
\Sigma\left(\mathcal{W} \backslash\left\{w_{n}\right\}, l\right) & =\Sigma(\mathcal{W}, l)-w_{n} \Sigma\left(\mathcal{W} \backslash\left\{w_{n}\right\}, l-1\right) \\
& =-w_{n} \Sigma\left(\mathcal{W} \backslash\left\{w_{n}\right\}, l-1\right) .
\end{aligned}
$$

Thus, in view of (3.3),

$$
\Sigma(\mathcal{W}, i)=0, \forall i \in\{1, \cdots, l\}
$$

implies

$$
\Sigma\left(\mathcal{W} \backslash\left\{w_{n}\right\}, l\right)=\left(-w_{n}\right)^{l}
$$

Then we have the following Theorem.

Theorem 6.1. Suppose $N=p q$ with $p$ and $q$ prime numbers and $K, 0<K<N$ is a multiple of $p$. Then there are $K$ distinct $N$-th roots of unity $w_{i}$, such that for each $m \in\{1, \cdots, p-1\}$, the m-products of $w_{i}$ form a vanishing sum.

Proof. Suppose for some $\alpha>0, K=\alpha p$. As $K<N, \alpha \in \mathbb{Z}_{q} \backslash\{0\}$. Choose for distinct $x_{i} \in\{1, \cdots, \alpha\} \subset \mathbb{Z}_{q}$,

$$
S_{p}\left(x_{i}\right)=x_{i}+q \mathbb{Z}_{p}
$$

Choose

$$
\mathcal{W}=\left\{w_{i}=e^{j 2 \pi \frac{s_{i}}{N}} \mid s_{i} \in \bigcup_{i=1}^{\alpha} S_{p}\left(x_{i}\right)\right\}
$$

As each $S_{p}\left(x_{i}\right)$ has cardinality $p$,

$$
\left|\bigcup_{i=1}^{\alpha} S_{p}\left(x_{i}\right)\right|=\alpha p .
$$

Further as the $x_{i} \in \mathbb{Z}_{q}$ are distinct so are the elements of this set. Finally, as all elements of this set are in $\mathbb{Z}_{N}$, the elements of $\mathcal{W}$ in (6.8) are distinct.

We now proceed to prove by induction that such a $\mathcal{W}$ obeys (6.3) for all $l \in$ 
452

$\{1, \cdots, p-1\}$. First observe that for all $l \in\{1, \cdots, p-1\}$

$$
\begin{aligned}
\sum_{i=1}^{\alpha p} w_{i}^{l} & =\sum_{k=1}^{\alpha} \sum_{n=0}^{p-1} e^{j 2 \pi l \frac{x_{k}+n q}{p q}} \\
& =\sum_{k=1}^{\alpha} e^{j 2 \pi l \frac{x_{k}}{p q}} \sum_{n=0}^{p-1} e^{j 2 \pi \frac{n l}{p}} \\
& =\sum_{k=1}^{\alpha} e^{j 2 \pi l \frac{x_{k}}{p q}} \frac{1-e^{j 2 \pi l}}{1-e^{j 2 \pi \frac{l}{p}}} \\
& =0,
\end{aligned}
$$

where the penultimate equation critically relies on the facts that $p$ is prime and $l \in\{1, \cdots, p-1\}$.

Substituting $l=1$ in (6.9) proves the result for $m=1$. Now suppose the result holds for all $m \in\{1, \cdots, p-2\}$. Then from (6.6) holds for $l=m$ and all $w_{n} \in \mathcal{W}$. Thus from (6.1) there holds:

$$
\begin{aligned}
(m+1) \Sigma(\mathcal{W}, m+1) & =\sum_{i=1}^{\alpha p} w_{i} \Sigma\left(\mathcal{W} \backslash\left\{w_{i}\right\}, m\right) \\
& =\sum_{i=1}^{\alpha p}\left(-w_{i}\right)^{m+1} \\
& =(-1)^{m+1} \sum_{i=1}^{\alpha p} w_{i}^{m+1} \\
& =0
\end{aligned}
$$

where we have used (6.9) and the fact that $m+1 \in\{1, \cdots, p-1\}$.

In view of Theorem 3.1 this thus shows that if $K$ is a multiple of $p$ then for all $1<m<p, A(\mathcal{S}(K, m), N)$ lacks full spark. A corresponding result also holds for multiple of $q$. The proof does not work for $m=p$ for example. This is so as in such a case (6.9) no longer holds. The next theorem provides a much broader class of $K$ for which $A(\mathcal{S}(K, m), N)$ lacks full spark.

Theorem 6.2. Suppose $N=p q$ with $p$ and $q$ prime numbers and $N>K>0$. Suppose $m \in\{1, \cdots, p-1\}$, and at least one member of $\{K-m+1, \cdots, K\}$, is $\alpha p, \alpha>0$. Then there are $K$ distinct $N$-th roots of unity $w_{i}$, such that the 
m-products of $w_{i}$ form a vanishing sum.

Proof. The result is clearly true for $m=1$. Thus consider $m>1$. Suppose for some $l \in\{1, \cdots, m-1\}, K-l$ is $\alpha p, \alpha>0$. From Theorem 6.1, there are $K-l$ distinct $N$-roots of unity all whose $n$-products form a vanishing sum for all $n \in\{1, \cdots, p-1\}$. In other words the set $\mathcal{W}_{K-l}=\left\{w_{1}, \cdots, w_{K-l}\right\}$ has the property that

$$
\Sigma\left(\mathcal{W}_{K-l}, n\right)=0, \forall n \in\{1, \cdots, p-1\} .
$$

As $K<N$, there are clearly $l, N$-th roots of unity that do not belong to $\mathcal{W}_{K-l}$. Call these $w_{i}, i \in\{K-l+1, \cdots, K\}$. Define $\mathcal{W}_{K-l+i}=\left\{w_{1}, \cdots, w_{K-l+i}\right\}$. We will use induction to show that for all $i \in\{0, \cdots, l\}$

$$
\Sigma\left(\mathcal{W}_{K-l+i}, n\right)=0, \forall n \in\{i+1, \cdots, p-1\}
$$

As $l<m$, this will prove the result. From (6.10), (6.11) holds for $i=0$. Now suppose (6.11) holds for some $i<l$. Then from (6.2), and all $n \in\{i+2, \cdots, p-$ $1\}$ and (6.11)

$$
\begin{aligned}
\Sigma\left(\mathcal{W}_{K-l+i+1}, n\right) & =w_{K-l+i+1} \Sigma\left(\mathcal{W}_{K-l+i}, n-1\right)+\Sigma\left(\mathcal{W}_{K-l+i}, n\right) \\
& =0
\end{aligned}
$$

Theorem 3.1, Theorem 6.1 and Theorem 6.2 effectively prove the following.

Theorem 6.3. Suppose $N=p q$ with $p$ and $q$ prime numbers and $N>K>0$. Suppose $n \in\{1, \cdots, p-1\}$ (respectively $n \in\{1, \cdots, q-1\}$ ), and at least one member of $\{K-n+1, \cdots, K\}$, is $\alpha p$ (respectively $\alpha q), \alpha>0$. Then $A(\mathcal{S}(K, n), N)$ does not have full spark.

This theorem precludes a vast swathe of cases from having full spark and plays the important role of excluding many sampling patterns. For example with $p=5$ and $q=11$, i.e. $N=55$. Then $A(\mathcal{S}(K, 8), N)$ does not have full spark for $K \in\{22,23,24,25,26,27\}$. Similarly, in Example $2.2 N=2 \times 5, K=4$ and $n=2$. While the example fails the sufficient condition of Theorem 5.3 as $\left(\begin{array}{l}K \\ n\end{array}\right)=6$ is a nonnegative multiple of the prime factor 2, the explanation of lack of spark is not conclusive from Theorem 5.3. However, Theorem 6.3 definitively provides the explanation as $K$ is a multiple of 2 . 


\section{Simulation Results}

We now provide several simulations. First we illustrate the negative results of Section 6 with nontrivial matrices whose spark would be computationally prohibitive to check. First consider $N=35=5 \times 7, n=2$ and $K=8$. As $K-1=7$ by Theorem $6.3 A(\mathcal{S}(K, n), N)$ does not have full spark. Indeed one can verify that rank of the $7 \times 7$ submatrix obtained by retaining columns indexed by multiples of 5 has rank 6 . Checking the spark of a $7 \times 35$ matrix requires checking $\left(\begin{array}{c}35 \\ 7\end{array}\right)=6724520$ submatrices.

More dramatically, consider the $38 \times 2183, A(\mathcal{S}(K, n), N)$ obtained by $N=$ $2183=37 \times 59, K=38$ and $n=2$. Theorem 6.3 correctly predicts that this cannot have full spark as $K-1$ is a multiple of a prime factor of 37 . Indeed the $38 \times 38$ matrix obtained by retaining columns that are multiples of 59 has rank 36 . Observe that to check the spark of this $A(\mathcal{S}(K, n), N)$ would require checking the rank of $\left(\begin{array}{c}2183 \\ 38\end{array}\right)>10^{82}$ submatrices of $A(\mathcal{S}(K, n), N)$.

Next we turn to implications of satisfying the condition in Theorem 5.3. To this end we examine the performance of $\ell_{1}$-recovery of a $k$-sparse vectors $x$ of length $N$ with Gaussian random entries from observations $y=A x$, where $A$ is the submatrix of the $N$-point DFT matrix obtained by selecting the first $K$ consecutive rows, with the row indexed by $n$ missing. For all cases we choose $N=37 \times 59, n=2$ and $K=36$. One can verify that $\left(\begin{array}{c}36 \\ 2\end{array}\right)=630$ is not a nonnegative integer combination of the two prime factors 37 and 59.

We assume that the observation vector $y$ is corrupted by noise to get $y_{n}=$ $y+\sigma \xi\|y\|_{2}$, where $\sigma\|y\|_{2}$ is the noise standard deviation and $\xi$ is a random vector of the same length as $y$ with elements drawn from $\sim C N(0,1)$.

The recovery of such a $x$ from a corrupted $y_{n}$ is shown for various sparsity levels in Figure 1. Specifically, recovery was effected as the constrained optimization

$$
\min \|x\|_{1} \text {, subject to }\left\|\mathbf{y}_{n}-A x\right\|_{2} \leq u\left\|\mathbf{y}_{n}\right\|_{2},
$$

using the Matlab's Disciplined Convex Programming, CVX routine. The recovery error in $x, \frac{\left\|x-x_{\text {estimated }}\right\|_{2}}{\|x\|_{2}}$ is drawn on the $\mathrm{y}$-axis, against the SNR on the $\mathrm{x}$-axis. SNR is defined as $10 \log \frac{\|y\|_{2}^{2}}{\left\|y_{n}-y\right\|_{2}^{2}}$. Each data point on the plot is obtained after averaging 50 realizations of $x$.

As by Theorem 5.3, the observation matrix has full spark 37, theoretically $\ell_{0}$ recovery with $k \leq 17$ is possible. However $\ell_{1}$ recovery should be possible only with a much smaller sparsity level. Yet at high SNRs even vectors with sparsity level of $k \in\{15,16,17\}$ are effectively recovered. 


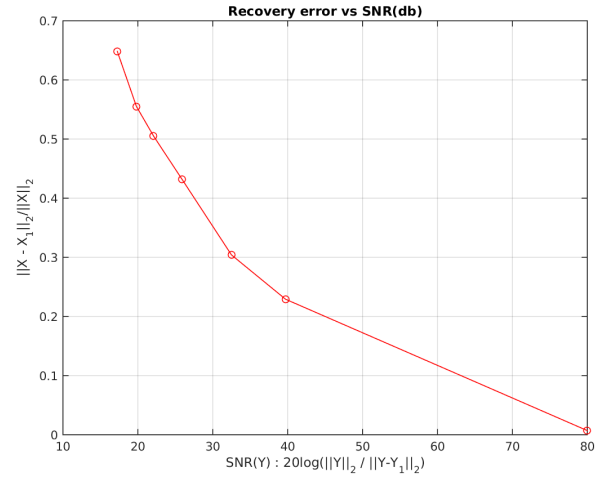

(a) $K=36$, sparsity level $k=6$

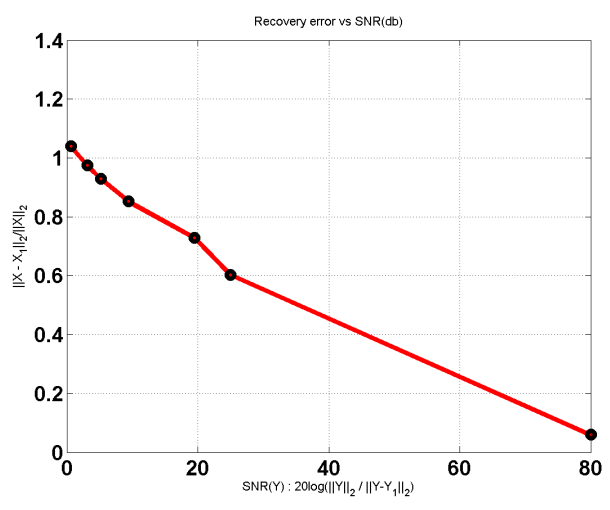

(c) $K=36$, sparsity level $k=16$

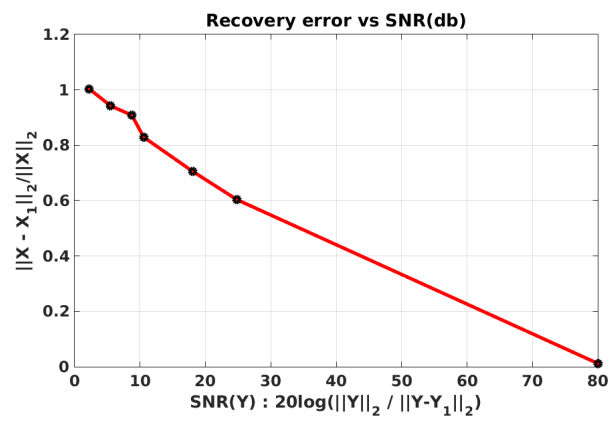

(b) $K=36$, sparsity level $k=15$

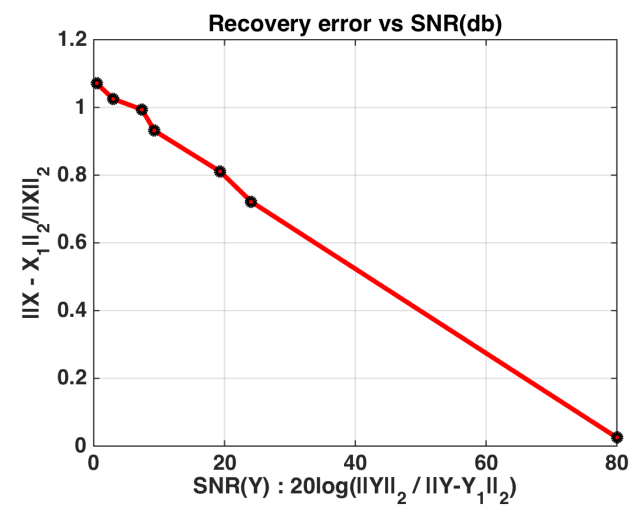

(d) $K=36$, sparsity level $k=17$

Figure 1: $l_{1}$ recovery error vs SNR for various sparsity levels.

\section{Conclusion}

We have addressed the following question: When is the submatrix of the $N \times N$, DFT matrix obtained by removing certain rows has full spark, when the rows that are retained are contiguous with precisely one missing. We have argued that because of Theorem 2.2, the full spark nature of such a matrix yields several others with the same property. We have established an equivalence between the full spark property of such matrices and the existence of roots of unity whose products, or themselves, form a vanishing sum. Some, necessary, some sufficient and some necessary and sufficient conditions on the prime factors of $N$, the number of rows that are retained and the index of the row whose absence prevents the set of retained rows from being completely contiguous. 


\section{References}

[1] P. Pal and P. P. Vaidyanathan, "Theory of Sparse Coprime Sensing in Multiple Dimensions", IEEE Transactions on Signal Processing, pp. 3592 - 3608, 2011.

[2] M. Lustig, D. L. Donoho, J. M. Santos, and J. M. Pauly, "Compressed sensing MRI," IEEE Signal Processing Magazine, vol. 25, no. 2, pp. 72-82, 2008.

[3] R. Chartrand, "Fast algorithms for nonconvex compressive sensing: MRI reconstruction from very few data," in IEEE International Symposium on Biomedical Imaging: From Nano to Macro, 2009. ISBI'09. IEEE, 2009, pp. 262-265.

[4] U. Gamper, P. Boesiger, and S. Kozerke, "Compressed sensing in dynamic MRI,” Magnetic Resonance in Medicine, vol. 59, no. 2, pp. 365-373, 2008.

[5] R. Baraniuk and P. Steeghs, "Compressive radar imaging," in Radar Conference, 2007 IEEE. IEEE, 2007, pp. 128-133.

[6] V. M. Patel, G. R. Easley, D. M. Healy Jr, and R. Chellappa, "Compressed synthetic aperture radar," IEEE Journal of Selected Topics in Signal Processing, vol. 4, no. 2, pp. 244-254, 2010.

[7] L. Zhang, M. Xing, C.-W. Qiu, J. Li, J. Sheng, Y. Li, and Z. Bao, "Resolution enhancement for inversed synthetic aperture radar imaging under low SNR via improved compressive sensing," IEEE Transactions on Geoscience and Remote Sensing, vol. 48, no. 10, pp. 3824-3838, 2010.

[8] D. L. Donoho and M. Elad, "Optimally sparse representation in general (nonorthogonal) dictionaries via 1 minimization," Proceedings of the $\mathrm{Na}$ tional Academy of Sciences, vol. 100, no. 5, pp. 2197-2202, 2003. 
[9] P. Feng and Y. Bresler, "Spectrum-blind minimum-rate sampling and reconstruction of multiband signals," in IEEE International Conference on Acoustics, Speech, and Signal Processing, 1996. ICASSP-96. Conference Proceedings., 1996, vol. 3. IEEE, 1996, pp. 1688-1691.

[10] E. J. Candes and T. Tao, "Near-optimal signal recovery from random projections: Universal encoding strategies?" Information Theory, IEEE Transactions on, vol. 52, no. 12, pp. 5406-5425, 2006.

[11] E. J. Candès, J. Romberg, and T. Tao, "Robust uncertainty principles: Exact signal reconstruction from highly incomplete frequency information," Information Theory, IEEE Transactions on, vol. 52, no. 2, pp. 489-509, 2006.

[12] Y. C. Eldar and G. Kutyniok, Compressed Sensing: Theory and Applications. Cambridge University Press, 2012

[13] T. Strohmer and R. W. Heath Jr, "Grassmannian frames with applications to coding and communication," Applied and computational harmonic analysis, vol. 14, no. 3, pp. 257-275, 2003.

[14] A. S. Bandeira, M. Fickus, D. G. Mixon, and P. Wong, "The road to deterministic matrices with the restricted isometry property," Journal of Fourier Analysis and Applications, vol. 19, no. 6, pp. 1123-1149, 2013.

[15] P. Xia, S. Zhou, and G. B. Giannakis, "Achieving the Welch bound with difference sets," IEEE Transactions on Information Theory, vol. 51, no. 5, pp. 1900-1907, 2005.

[16] N. Y. Yu and Y. Li, "Deterministic construction of Fourier-based compressed sensing matrices using an almost difference set," EURASIP Journal on Advances in Signal Processing, vol. 2013, no. 1, pp. 1-14, 2013.

[17] B. Alexeev, J. Cahill, and D. G. Mixon, "Full spark frames," Journal of Fourier Analysis and Applications, vol. 18, no. 6, pp. 1167-1194, 2012.

[18] T. Lam and K. Leung, "On vanishing sums of roots of unity," Journal of algebra, vol. 224, no. 1, pp. 91-109, 2000.

[19] G. Sivek, “On vanishing sums of distinct roots of unity," Integers, vol. 10, pp. 365-368, 2010. 
[20] P. Stevenhagen and H. W. Lenstra, "Chebotarëv and his density theorem," Math. Intelligencer, vol. 18, pp. 26-37, 1996.

[21] P. P. Vaidyanathan, Multirate Systems and Filter Banks. Prentice Hall, 1993.

[22] H. K. Achanta, S. Biswas, S. Dasgupta, M. Jacob, B. N. Dasgupta and R. Mudumbai, "Coprime conditions for Fourier sampling for sparse recovery", in Proceedings of IEEE 8th Sensor Array and Multichannel Signal Processing Workshop (SAM), pp. 533-536, DOI: 10.1109/SAM.2014.6882460, A Coruna, Spain, June 2014.

[23] M. E. Dominguez-Jimenez, N. Gonzalez-Prelcic, G. Vazquez-Vilar, and R. Lopez-Valcarce, "Design of universal multicoset Ssampling patterns for compressed sensing of multiband sparse signals," in Proceedings of IEEE International Conference on Acoustics, Speech and Signal Processing (ICASSP), pp. 3337-3340, DOI: 10.1109/ICASSP.2012.6288630, Kyoto, 2012. 